\title{
Optimizing ICT Resources to Mitigate Contagion: A Case Study of COVID-19 Pandemic
}

\author{
James Kunle Olorundare ${ }^{1,}$, , Oluwafemi Emmanuel Ekanoye ${ }^{2}$, Adebimpe Olubunmi Olorundare ${ }^{3}$ \\ ${ }^{1}$ Technical Standards and Network Integrity, Nigerian Communications Commission, Abuja, Nigeria \\ ${ }^{2}$ Office of Governmental Contracting and Services, Southern University and A\&M College, Louisiana, USA \\ ${ }^{3}$ Information Technology Department, National Open University of Nigeria, Abuja, Nigeria
}

\section{Email address:}

olorundarek@ncc.gov.ng (J. K. Olorundare), oluwafemi.ekanoye@sus.edu (O. F. Ekanoye), femiekanoye125@gmail.com (O. F. Ekanoye), bolorundare@gmail.com (A. O. Olorundare)

${ }^{*}$ Corresponding author

\section{To cite this article:}

James Kunle Olorundare, Oluwafemi Emmanuel Ekanoye, Adebimpe Olubunmi Olorundare. Optimizing ICT Resources to Mitigate Contagion: A Case Study of COVID-19 Pandemic. International Journal of Science, Technology and Society.

Vol. 9, No. 3, 2021, pp. 135-141. doi: 10.11648/j.ijsts.20211003.14

Received: December 3, 2020; Accepted: December 10, 2020; Published: June 16, 2021

\begin{abstract}
COVID-19 is ravaging many countries and there are unprecedented challenges in managing the pandemic. Basically, two strategies are being adopted; Traditional Health System and Telehealth which include telemedicine, remote monitoring of patient, Use of Artificial Intelligence (AI) etc. This paper discusses how the communications and computing resources are being used in the telehealth sector to respond to COVID-19. It expatiates the positive impact of telehealth on the general approach of managing COVID-19 pandemic and how this is rapidly facilitating flattening the curve of COVID-19 pandemic as seen in the country experiences of China and South Korea discussed. The research includes both the Secondary and Primary data. The literature review exhibited the secondary data conducted on this subject matter which was also validated using the quantitative survey conducted for the primary data collection. Experiences of selected countries were also discussed as case studies on how ICT resources are being deployed in the COVID-19 pandemic as practical evidence to the research. Recommendations were organically extracted from the result of the primary research data to fill the gap noticed in mitigating pandemic by optimizing ICT resources and infrastructures. The use of Artificial Intelligence, Robotics, emergency numbers for different applications and increase use of those ICT infrastructures and resources have assisted in mitigating COVID-19 in the illustrated examples. This has validated the results of the research and therefore given empirical evidence to the feasibility of the recommendations.
\end{abstract}

Keywords: Mobile Technology, Information and Communications Technology (ICT), Telehealth (EHealth or E-health), Mobile App, Artificial Intelligence (AI), Coronavirus Disease (COVID-19), Pandemic

\section{Introduction}

ICT infrastructure can be used to improve our healthcare system through the deployment of electronic health system on Information and Communication Technology platform as it is now being used during the COVID-19 pandemic either directly as in telehealth or indirectly as in such applications like telecommuting, e-commerce etc. Never before has ICT infrastructures been so critical to our society day- to-day activities. Routines such as general safety to mitigate COVID-19, health management, commercial activities etc are few examples of how communications networks and other IT infrastructures are adding values to our health sector. With the pressure on the health system. The ICT resources are being optimized for higher efficiency in order to fast track the mitigation of COVID-19.

The issue of social distancing and stay-at-home to prevent the spread of COVID-19 are now being facilitated by the use of ICT infrastructures. A survey conducted to find out how ICT resources can facilitate the suppression of COVID-19 was conducted and analyzed. The result discussed below showed that ICT tools are key in combatting COVID-19 pandemic. 
The objective of the paper is to show how ICT resources can be used to mitigate COVID-19. The motivation stemmed from the need to find alternative way to suppress COVID-19 as the traditional medical infrastructures are being overstretched all over the world. The scope of the paper is quite global since COVID-19 is a global pandemic and the survey conducted cut across many continents.

The paper from this point is arranged as follows: Literature review which is followed by methodology and data analysis followed by country experiences of selected countries based on unique scenarios in which specific strategies of ICT resources benefits are analyzed. These include, Telecommuting, E-commerce, Telehealth and others. Recommendations are made in section $\mathrm{V}$ which is followed by conclusion and future research work in section VI.

\section{Literature Review}

The COVID-19 pandemic has spread to many countries on the surface of the earth and the traditional health system of the world is being tested to its full capacity. There is a strong need to reduce the stress on the traditional health system. One of the promising techniques is the use of Information and Communication Technology to boost the performance of the health systems of many countries in terms of electronic health system or eHealth. EHealth is the deployment and application of Information and Communication Technology (ICT) for health and to optimize the health system. The eHealth can be said to be capacity expansion of the already existing traditional health system by the applications and optimization of ICT infrastructures. However, many countries have not really looked at how ICT resources can be optimized for mitigation in pandemic situation like COVID-19. And this is the gap the research intends to fill since COVID-19 pandemic is novel.

"Optimizing ICT resources to mitigate contagion: A case study of COVID-19 Pandemic" is the theme of this paper which came up as a result of COVID-19 pandemic currently ravaging the world (year 2020) and the need to get rid of the pandemic. The traditional health system cannot handle this pandemic without the use of ICT resources to facilitate the rate of mitigation because of the contagious nature of the pandemic.

ICT resources are being used for so many applications including eHealth. In order to get better result from using ICT resources to mitigate COVID-19 pandemic, it is important that the resources are optimized for increased efficiency. Contagion is "a disease that can be spread by people touching each other" [1].

The objectives of eHealth in each country are to promote and strengthen the use of ICT in health development from field applications to hospital procedure applications which can also include inter-state or interregional hospital connectivity and usage [2]. E-Health (it is the same as eHealth) is a new healthcare strategy which is still a developing practice that can only be achieved through the use of electronic processes and communication technologies [3]. E-health cannot be implemented without ICT resources. Hence, the baseline of applying eHealth is availability of ICT resources. And this can be excellent resources during pandemic and the question this research would answer is how ICT resources can be optimized to mitigate COVID-19 pandemic.

There exists an appreciable development in health Information Technology (IT) especially with respect to sharing of information due to the seamless presence of the Internet which makes it easy and also, broadband technology has brought the use of telemedicine which makes it possible for experts to be able to attend to patient in another location. This is a borderless service based on the use of Information and Communications Technologies [4]. Telemedicine would be an excellent tool to be harnessed against COVID-19 pandemic which is already taking its toll on the medical practitioners because of many infected people to attend to in the hospital.

Digital divide between the developed and the developing countries of the world is visible. This can be seen in the distribution of benefits from ICT that created global imbalance. For instance, United States of America and China account for $75 \%$ of all patents related to Blockchain technologies, $50 \%$ of global spending on IoT, $75 \%$ of the cloud computing market in addition to that, about $90 \%$ of the market capitalization value of the world's 70 largest digital platforms. This implies that there is huge digital divides in the world and half of the world population is still offline. This can be seen in the availability of communications infrastructures like broadband facilities and this digital divide also translate into the availability of telemedicine facilities and other technologies that can be used in the treatment of COVID-19 [5]. Regardless, ICT resources are needed to compliment traditional health system to combat COVID-19. How to optimize the ICT resources for this purpose will be discussed.

In a pandemic situation like COVID-19, eHealth in general, and telemedicine in particular can be a vital resource to remote regions of emerging and developing countries but it is often challenging to establish because of inadequate ICT infrastructure [5] For instance, in the early days of COVID-19 pandemic Nigeria was trying to set up more laboratories to test samples and limited availability of reagent was also a challenge.

The difficult financial situation in many African states implies that the majority of the African countries are poorly treated medically. In many developing countries, it is not only about insufficient facilities and trained manpower, but lack of accessibility to eHealth because of inadequate broadband facilities and access to the Internet access in unserved villages [6]. This is a serious challenge in time of COVID pandemic. There is need to devise strategy to optimize the available ICT resources. Developed countries are also grappling with coping with the pandemic because of the rate of infection and it is only feasible to rely on optimizing communications and telecommunications infrastructure to flatten the effect of COVID-19. This is what this paper intends to establish and proffer harmonized strategic solutions.

Self-monitoring is the use of sensors or tools which are readily available to the general public to track and record personal data. The sensors are usually wearable devices and 
the tools are digitally available through mobile device applications. From a survey conducted and reported by Olorundare et al, majority of Nigerians would really want self-monitoring system which is a good development in time of pandemic like the COVID-19. This implies that if health application is deployed using IoT monitoring systems, it will be embraced and this will help to reduce the rate of infection and in a short time, the rate of infection will be flatten through self-distancing and stay at home while those under quarantine can still be monitored without further exposing the health workers managing the sick. This is one of the advantages of self-monitoring system based on eHealth [7].

Telecoms operators and other ICT service providers are recognizing that their customers will rely on ICT services during stay-at-home in order to flatten the rate of spread; self-isolation for those that have contacted COVID-19 and unconfirmed cases still being tested and quarantine for those with confirmed COVID-19 cases. The overarching objectives are for working at home, personal or family entertainment and maintaining social connections with others. Also, for accurate information broadcast to all.

Operators in countries like France, Australia, Saudi Arabia, Bahrain, Russia and Belgium for the purpose of illustration have started working on an increasing or removal of data caps, or even free unlimited Internet especially for fixed subscribers [8]. The importance of this is for their citizens that are subscribers to those operators services to be seamlessly connected so that they can communicate in time of emergency for medical crisis or for them to be able to do away with boredom in terms of entertainments or to be able to do electronic transaction for the purpose of receiving or transferring money or to buy essentials online like facemask, food, groceries etc. This paper intends to itemize strategies on how ICT resources can be optimized for the purpose of mitigating COVID-19 pandemic.

\section{Methodology and Data Analysis}

\subsection{Research Methodology}

Secondary data are the already existing data collected by the investigators and this was collected and reviewed under the literature review in Section 2 above. It shows the relevant research works to the theme being researched the existing gap and to advance this work, the researchers conducted primary research using Quantitative Survey technique which was responded to within a week because it was conducted in the middle of COVID19 pandemic April 2020. The Primary data collection source for this research was collected through a survey at [9].

\subsection{Survey Questions, Infographics and Analysis}

(1) A survey of 10 key research questions was carried out in April 2020 and there were 100 responses in less than a week based on the trendy nature of the survey. Out of 100 respondents 87 respondents are from Nigeria and other parts of the world also responded except Australia with no response because of the random selection of respondents. The pie chart below Figure 1. represents the proportional distribution of the respondents.

\section{Chart Title}

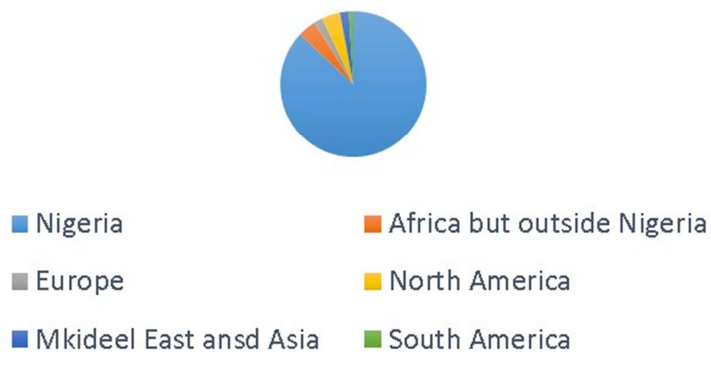

Figure 1. A Pie Chart showing distribution of 100 respondents.

(2) What is your educational level? The Bar Chart in Figure 2 below depicts the education level distribution of the respondents. It shows that $99 \%$ of the respondents have at least University degree which shows that they can understand the survey being answered correctly. This implies that the survey is valid and the result can be relied on because the respondents understand the survey.

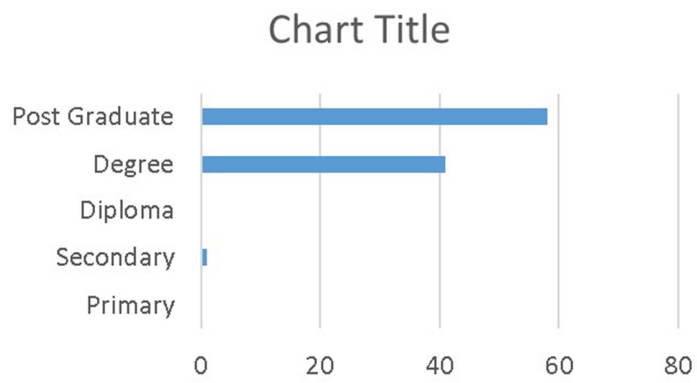

Figure 2. A Bar Chart showing the level of education for the respondents.

(3) Do you know about COVID-19 pandemic? This is a question to confirm if the respondents have fundamental knowledge of the survey. 97 out of 100 respondents know about the COVID-19 Pandemic which shows that the survey can be relied on and the responses are valid.

(4) Do you know about Information and Communications Technologies, and their applications? This deals with how to what extent the respondents are familiar with Information and Communication Technology and what it can be used for. The results showed that majority of the respondents have good knowledge of ICT as shown in Bar Char in Figure 3 below.

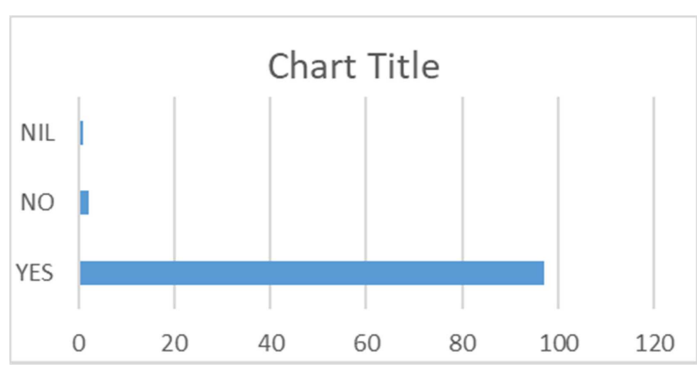

Figure 3. Bar Chart showing the answer to knowledge of ICT.

(5) Do you know that Robotics, Artificial Intelligence 
and other emerging technologies can be used to reduce the exposure of medical practitioners to COVID-19 contagion? $86 \%$ of the respondents answered in affirmative while $13 \%$ answered no and one respondent skipped. With this results majority of the respondents know that Robotics or Artificial Intelligence can be used in one way or the other to help in combatting COVID-19 contagion. The use cases in Section 4 below established and confirmed the knowledge of the majority of the respondent in this key research question.

(6) Can e-commerce (online store where you can buy food and other essentials) help to maintain, stay-at-home order, quarantine, isolation, during COVID-19 contagion? 97\% of the respondents answered in affirmative and $2 \%$ answered in Negative and $1 \%$ did not answer, This is valid and based on what is obtainable during the COVID-19 contagion, the answer confirmed that e-commerce is one of the ICT channels that can facilitate effective isolation, stay-at-home order which can help to combat contagion like COVID-19. The illustration in section 4 on China and South Korea experiences can also point to the validity of the survey.

(7) Can national emergency number information center, mobile network SMS broadcast, national agencies social media information dissemination help in educating the citizenry and broadcasting correct information on COVID19 hence, reduced misinformation in the public space? From the table below (Table 1), 89\% responded in affirmative to the question showing that majority are on the side that ICT tools like emergency number information center; Short Message Service (SMS) broadcast by Mobile Network Operator to give current and accurate information; Social Media pages wall and twitter handles of national agencies giving timely and accurate information can help in as much as one of these facilities can be accessed either in well-served or underserved area. However, unserved areas exist but the number of totally unserved places could be minimal and that can be explained from variance in answer like both, "Yes to a certain extent" and "No" can also come up in such areas as answers. Looking at the distribution of the answers, the frequency of "yes" shows that the above ICT media/channels would help a great deal if deployed.

Table 1. Table showing distribution of answer to Question 7 above.

\begin{tabular}{lll}
\hline S/N & RESPONSE & FREQUENCY \\
\hline 1 & NO & 3 \\
2 & YES & 89 \\
3 & BOTH & 1 \\
4 & NO ANSWER & 6 \\
5 & YES, CERTAIN EXTENT & 1 \\
\hline
\end{tabular}

(8) Can ICT applications such as Internet of Things, Robotics, Artificial Intelligence, Telecommunications Networks, Drones (UAV) applications help in reducing rate of COVID-19 infection propagation? Figure 4 below shows the distribution of answers given by respondents. The descending order showed that ICT applications like IoT, Artificial Intelligence, Drones etc can help in reducing
COVID-19 infection propagation through different applications. The illustration in Section 4 below is a validation of the majority answer to the survey and using pareto chart for grouping the frequency: "A lot" can be added to "A great deal" and "moderate" that is at the center of pareto chart, can be neutral and "Non at all", "No answer" and "a little" can be grouped on the negative. This gives a very large percentage of affirmative as shown in pareto chart Figure 4 below. The importance of pareto chart is to group answers in a progressive order and that has been established.

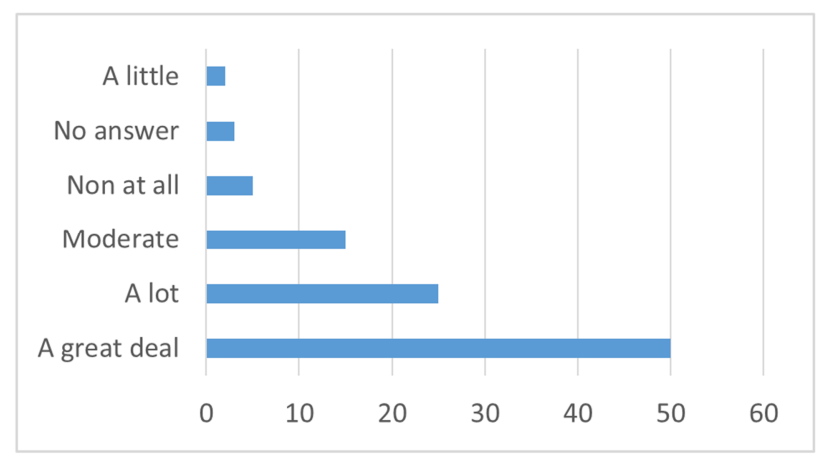

Figure 4. A Pareto Chart showing the distribution of responses in descending order of frequency.

(9) If we work from home based on Stay-at-Home order, can that help to reduce rate of COVID-19 infection propagation? Figure 5 below is a pie chart showing that $96 \%$ of the respondents agreed with the fact that working from home is part of the solution to reduce COVID-19 infection propagation. To work from home, there is need for robust Internet system which will connect us with our colleagues and other staff from the same office for proper organization and job process flow. Also, the use of mobile phone which is also part of the ICT tools is very key to being able to work from home effectively. This implies that the use of telecommunications networks, Internet and other ICT tools like laptops, desktop computers which are connected on the Internet either from direct access, Virtual Private Networks, intranet, extranet or any other type of connection is an integral part of working from home. Hence, this will help to combat COVID-19 contagion going by the large percentage of affirmative answers to "working from home" as recorded in the survey. Figure 5 below shows the answer to this survey question.

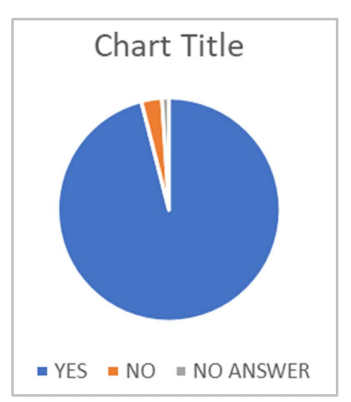

Figure 5. A Pie Chart showing that majority of respondents agreed with Working from home as a solution to COVID-19 infection Propagation.

(10) In your own word, how do you think ICT resources 
can be optimized to reduce COVID-19 infection propagation rate?

There are 88 free comments made and up to $70 \%$ agreed through different media that the following can help:

\subsection{ICT Infrastructures}

i. Internet of Things applications and smart systems

ii. Artificial intelligence applications

iii. E-commerce and telecommuting

iv. Mobile Telephony

v. Telemedicine

vi. Broadband facilities

vii. Drones

viii. Policies on Electronic activities like E-commerce, Telecommuting etc

All these point to the fact that optimization of ICT tools and infrastructures can help to combat pandemic like COVID-19 contagion directly or indirectly and the illustration of country experience below also established this valid point.

\section{Country Experience to Illustrate Optimization of ICT Resources}

\subsection{China COVID-19}

The Chinese government used strategy of Quick Responses together with Intense and Rapid Contact Tracing. In addition to surveillance of potential contacts and Isolation. But the COVID-19 pandemic rate of infection was high and e-Health based on ICT infrastructures were applied as explained below [10]. This is a validation of the quantitative survey analyzed in section III above.

A. The country implemented large-scale contact tracing in the early 2000s during the SARS epidemic. China learnt from SARS of 2002-2003 by setting up large-scale surveillance system which can implement contact tracing by identifying and tracking people who may have been in contact with an infected person. The system is "idiot-proof" and if a tracer makes a mistake on questionnaire or forms, the screen would flash a yellow color alert on the screen [10].

B. China used technology that aims to trace every single COVID-19 case.

The Emergency centers in china is used to track the virus using huge screen to display the critical cluster of the virus for proper surveillance, monitoring and to arrest the situation. This is in addition to Chinese social media including Weibo (a microblogging website and app which compares to twitter and Instagram), Tencent (specializes in social networking, music, web-portals, e-commerce, mobile games and other internet services) and WeChat (a multi-purpose messaging social media and mobile payment app) were used to broadcast and share current and accurate information [10]. This validated and established Question 7 of the survey conducted and analyzed in section III above.

C. The country postponed non-urgent medical care and moved many doctor's visits online.

The Chinese model include prioritization of critical health issues. And the non-critical ones are given less priority and moved online. These include most of the normal physical routine to be able to keep regular services going, prescriptions in an orderly manner. And this gave room for concentration on COVID-19. It helps to also solve the problem of overcrowded medical systems which is affecting most of the countries with COVID-19 [10]. It also helped to reduce the exposure of the medical practitioners to COVID19. It is also a validation of Question 5 in the analyzed survey in section III above.

D. Online Shopping

In China, practical barriers to staying put in order to prevent spreading or contracting the illness were largely eliminated using the online system like buying fresh food online, groceries and other essentials. This help in suppressing the spread and to also maintain the social distance by staying at home. This is also good for isolation cases and quarantine.

This also helped in reducing panic buying and supplies because e-commerce helps in bringing the essentials to customer's doorstep steadily [10].

E. Telecommuting

In the past, many people did not want to discuss this but due to COVID-19 pandemic and the need to stay out of office many people in industries who did not want to implement it are now advocating for it and also working from home. Industries such as banking, aerospace engineering industry to the teaching profession in America. Telecommuting extends to spending time with friends and family on video calls as well in order to prevent boredom and sense of isolation [11]. Telecommuting can increase productivity; boost morale and it can be less stressful. These are some of the benefits of telecommuting among others.

One of the effective strategies to suppress the spread of COVID-19 is working from home while people are isolating or in quarantine. Working from home is made possible through the use of ICT resources connected through the Internet and associated technologies like Internet of Things which can use a monitoring module connected to the internet to monitor if the work is being done or not. Video conferencing can also be done for executive meeting and departmental routine meeting. For schedules that can be done from home this was implemented. The flipside of this is that there are some schedules that may not be online like logistics and that may have to be dealt with in a different way. COVID-19 has shown the need to work from home when it is practicable as shown from the China COVID-19 experience. Other part of the world has started using a similar strategy to suppress the spread of COVID-19 [12, 13]. This is a validation of the result got from Question 9 of the survey in section III above.

\subsection{South Korea: Use of ICT Tools to Suppress COVID-19}

A. Fast-developed testing kit: Combatting COVID-19 goes beyond the use of traditional medicine. Innovations and other initiatives beyond social distancing, need to be employed to 
rapidly flatten the curve. To flatten the curve, South Korea has used Artificial Intelligence to develop test kits within a very short time of 3 weeks and South Korea has a testing capacity of 15,000 per day on average. This has helped to flatten and bring down the curve rate of infection [14]. This validates the results of Question 8 of the survey in section III.

B. Smart quarantine information system: This is a system that allows the monitoring of incoming passengers from epidemic countries and this information is shared with relevant agencies (Ministry of Foreign Affairs, Health Insurance Review \& Assessment Service, telecommunication companies and the Korea Centers for Disease Control and Prevention KCDC) in Korea. The passengers are monitored within the incubation period of the disease the system is an ICT platform which uses roaming data of oversea arrival [15].

C. Mobile phone technology data for contact tracing and Mobile App for Information Sharing: Several mobile apps have been developed in a very short time which can show where to buy mask, another one can direct a patient to the closest testing center [14]. There are other one that give public critical information and so many applications. These help to combat COVID -19 and to slow down the rate of infection which was initially rapid

D. AI for improving diagnosis efficiency and patient classification: The South Korea has developed a Chest X-Ray AI Image Support Decision Tool. This helps to categorize the cases of COVID-19 into Mild, moderate, severe and very severe, this is a risk mitigation strategy which helps in treating each case based on the category. The system is based on an algorithm for identifying abnormal findings on chest $\mathrm{x}$ rays. The systems can examine the lungs within just three seconds [14]. This further validates question 8 results from section III survey.

E. Daegu: Making use of a smart city hub: Daegu was the epicenter of COVID-19 in South Korea but it is now being transformed into a Smart City and the Smart Hub in the city has been used during the epidemiological investigation to create route tracing application being used to develop new medicine [14].

\section{Recommendations}

From the foregoing, the following recommendations are hereby made:

1) ICT Pandemic Management System: Based on the foregoing, it is recommended that, there is need to set up ICT Pandemic Management System (ICT PMS) which will be used during Pandemic situation like the COVID19 by each country. This will include the strategy to work online and to monitor staff by manager and at the same time to make them feel under office environments but not making the staff feel uncomfortable. Applications that can handle such activities are already developed and being advanced based on lesson learnt from COVID-19. The app would also help workers to overcome boredom since they can see other staff working through their webcams on the screen face slate. This is an all-inclusive solution that can even help managers to help to monitor duration of staff work.

2) It is expected that this will include Business Continuity Management ISO22301 that can be used to define management system policy for all climes/countries and this should be expanded to include how countries can combat pandemic such as COVID-19 by including robust policy on Artificial Intelligence to combat future occurrence. This can be a policy document processed through the Act of Parliament with unique definitions for each country as deemed fit. This will accelerate the optimization of ICT resources to combat contagion.

3) Emergency number: Setting up of national emergency number should be prioritized for countries that have not set up such facilities for the purpose of communications for victim or infected people to be able to communicate with the right channel like ambulance service, direction to close test center, direction to controlled market to buy Personal Protection Equipment (PPE), how to get drone services and what it can deliver, Disease Control Agency etc. this should be all inclusive robust platforms. This is a point to note for developing countries to implement a robust emergency platform. This validates the result of the survey in section III as established in country experience analyzed in section IV.

4) The use of Artificial Intelligence, Augmented Reality and smart systems for monitoring people for the purpose of tracking them in terms of movement should be set up by all countries so that tracking and tracing contacts would be easier. This can be implemented through Smart Hub, IoT, Big Data Analytics applications. Instead of the manual tracing which may not yield the desired results in case of larger samples like the COVID-19 in some countries that have been seriously affected. The country experience model above which can help to locate concentration of highly infected area integrated with "idiot -proof" system can be advanced by further applications of internet of things to integrate more monitoring systems. It is a known fact that for novel diseases and contagion it takes time to study the full epidemiology, to sequence the genome and to produce drugs and vaccine within the period of contagion before medical breakthrough, Artificial Intelligence can also be deployed to suppress and combat the contagion. South Korea and China illustration demonstrated this and validated questions 5, 7,8 and 10 of the survey analyzed in section III above.

5) For effective optimization of ICT resources, it is recommended that framework or policy on telehealth should also be considered as a standalone policy. This will define the minimum resources require to establish a telehealth for a particular country. The definition of what is expected in the telehealth services can also be included. It is expected that the minimum resources required for implementation like broadband standards be included. This should define the hardware requirements as it were. 
6) Work should commence on the implementations of the above recommendations as a way of preventing or combatting future contagion.

\section{Conclusion and Future Research Work}

The COVID-19 pandemic rate of infection is high and it is a fact that the traditional health system capacity needs to be boosted using the ICT resources in terms of electronic health system running on the ICT resources. The country experiences of South Korea and China have shown that without optimizing available ICT resources to do more in emergency situation like the COVID-19 pandemic, it will be difficult to slow down the rate of infection. Use of Artificial Intelligence, Robotics, emergency numbers for different applications and increase use of those ICT infrastructures and resources have assisted in mitigating COVID-19 in the illustrated examples. This has validated the results of the research and therefore given empirical evidence to the feasibility of the recommendations.

\section{Declaration}

Future research work shall be: "Regulation and Standardization of Digital Technologies Used in Facilitating the Mitigation of a pandemic disease".

\section{References}

[1] A. S. Hornby, D. Lea and J. Bradbery, Oxford advanced learner's dictionary of current English, Oxford University Press, $10^{\text {th }}$ edition ed. 2020.

[2] World Health Organization, "EHealth at WHO", ehealth Unit Geneva, Switzerland. [Source: online]. Available: https://www.who.int/ehealth/about/en/, [Accessed: 12th April, 2020].

[3] D. Mea, Vincenzo, "What is e-Health (2): The death of telemedicine?". Journal of Medical Internet Research. 3 (2): e22. [doi: 10.2196/jmir.3.2.e22. PMC 1761900. PMID 11720964., 2001].

[4] O. S. Daudi and M. Mughwira, Application of ICT in strengthening health information systems in developing countries in the wake of globalization, ACTS [African Centre for Technology Studies, pp 194-198., 2004] Source: online] Available:

https://www.ncbi.nlm.nih.gov/pmc/articles/PMC2688333/

[Accessed: 12th April, 2020].

[5] United Nations Conference on Trade and Development, Digital Economy Report 2019 Value Creation and Capture: Implications for Developing Countries, [United Nations Publications, Geneva., 2019].
[6] G. Bethscheider, (2015). Barney, Randall (ed.). "Satellite is vital for a unified, global, E-Health system. An SES Techcom Services Perspective". World Teleport Association. [Source: online]. Available: https://www.worldteleport.org/news/249029/Satellite-Is-VitalFor-A-Unified-Global-e-Health-System...-An-SES-TechcomServices-Perspective-.htm [Accessed: $12^{\text {th }}$ April, 2020].

[7] O. J. Kunle, O. A. Olubunmi and S. Sani, Internet of things prospect in Nigeria: Challenges and solutions, IEEE 3rd International Conference on Electro-Technology for National Development [NIGERCON, Owerri, pp. 736-745. 2017].

[8] International Telecommunications Union, (2020) Telecoms, Coronavirus and keeping the networks running: Opinion Available: online] Source: https://news.itu.int/telecomscoronavirus-and-keeping-the-networks-running-time-forleadership/ [2020] [Accessed: 12th April, 2020].

[9] Survey Monkey, [Source: online] Available: https://www.surveymonkey.com/r/V3YDQLH under the SurveyMonkey application. [Accessed: April, 2020].

[10] H. Brueck, 11 extreme measures China took to contain the coronavirus show the rest of the world is unprepared for COVID-19. [Source: online] Available: https://www.pulse.com.gh/bi/tech/11-extreme-measureschina-took-to-contain-the-coronavirus-show-the-rest-of-theworld/he13wmg [2020] [Accessed: 7th April, 2020]

[11] B. Morgan, Is COVID-19 Forcing Your Digital Transformation? 12 Steps To Move Faster, Forbes. 2020. [Source: online] Available: https://www.forbes.com/sites/blakemorgan/2020/04/05/iscovid-19-forcing-your-digital-transformation-12-steps-tomove-faster/\#56f55df3617b [Accessed: 12th April, 2020].

[12] S. Banjo, L. Yap, C. Murphy and V. Chan, The Coronavirus Outbreak Has Become the World's Largest Work-From-Home Experiment. Time. 2020. [Source: online] Available: https://time.com/5776660/coronavirus-work-from-home/ [Accessed: 12th April, 2020].

[13] A. Holmes, Employees at home are being photographed every 5 minutes by an always-on video service to ensure they're actually working — and the service is seeing a rapid expansion since the coronavirus outbreak, Business Insider by Pulse. 2020 [Source: online] Available: https://www.pulse.com.gh/bi/tech/employees-at-home-arebeing-photographed-every-5-minutes-by-an-always-onvideo/9bdc24p [Accessed: 10th April, 2020].

[14] International Telecommunications Union, COVID-19: How Korea is using innovative technology and AI to flatten the curve 2020. [Source: online]. Available: https://news.itu.int/covid-19-how-korea-is-using-innovativetechnology-and-ai-to-flatten-the-curve/ [Accessed: 7th April, 2020].

[15] Osong, Health Technology Administration Complex, Smart quarantine information system project, KCDC, Korea. 2019. [Source: online]. Available: http://www.cdc.go.kr/contents.es?mid=a30301180000 [Accessed: 15th April, 2020]. 